\title{
Memory effect on the formation of drying cracks
}

\author{
Michio Otsuk:* \\ Department of Pure and Applied Sciences, University of Tokyo, Komaba, Tokyo 153-8902, Japan
}

(Dated: April 5, 2018)

\begin{abstract}
We propose a model for the formation of drying cracks in a viscoplastic material. In this model, we observe that when an external force is applied to a viscoplastic material before drying, the material memorizes the effect of the force as a plastic deformation. The formation of the drying cracks is influenced by this plastic deformation. This outcome clarifies the result of a recent experiments which demonstrated that a drying fracture pattern on a powder-water mixture depends on the manner in which an external force is applied before drying. We analytically express the position of the first crack as a function of the strength of an external force applied before drying. From the expression, we predict that there exists a threshold on the strength of the force. When the force applied is smaller than the threshold, the first crack is formed at the center of the mixture; however, when the force applied exceeds the threshold, the position of the first crack deviates from the center. The extent of the deviation increases as a linear function of the difference between the strength of the force and the threshold.
\end{abstract}

PACS numbers: 46.50.+a, 83.60.La, 46.35.+z

\section{INTRODUCTION}

Cracks are observed on various materials such as rocks, tectonic plates, and paintings. These cracks are fascinating and has been studied by many researchers. The study of drying cracks in a powder-water mixture was also included in these researchers. In one example, it was noticed that when a layer of a powder-water mixture is dried in a container, it shrinks and cracks are formed on it [1]. The cracks extend from the surface of the mixture to the bottom and propagate horizontally along a line. As a result, a two-dimensional fracture pattern is formed on the surface of the mixture.

When we gently pour a powder-water mixture into a container and leave it undisturbed during the drying process, a random, isotropic fracture pattern is formed. However, Nakahara and Matsuo reported that when an external force is applied to the mixture before drying, the fracture pattern changes depending on the manner in which the force is applied 22. For example, when the mixture is vibrated in one direction before drying, cracks that are perpendicular to the direction of the vibration emerge first. Finally, a lamellar fracture pattern is formed. It takes more than 3 days for the cracks to be formed after the vibration. It is quite surprising that the effect of applying the force remains for such a long time.

The experimental result attained by Nakahara and Matsuo represents that a fracture pattern is controlled by the memory of an external force applied before drying. Similar memory effects that a response can be controlled by the memory of an operation have been observed in other materials, such as sand piles [3], micro-gel pastes [4], and rubbers [5]. By recalling that these memory effects have been studied from a rheological point of view,

*Electronic address: otsuki@jiro.c.u-tokyo.ac.jp we conjecture that the rheological property of a mixture plays an important role in the memory effect on the formation of drying cracks.

Among the rheological properties of the mixture, the most conspicuous one may be plasticity. Hence, we study the role of plasticity in the memory effect on the formation of drying cracks. First, in Sec. III we propose a model of the formation of drying cracks in a viscoplastic material. In Sec. III we find that a viscoplastic material memorizes the effect of an external force before drying as a plastic deformation by calculating the model numerically. By the influence of this plastic deformation, when an external force is applied to the material, a crack perpendicular to the force emerges earlier than when no force is applied. Based on this result, we conjecture that the perpendicular cracks emerge first by a plastic deformation. Furthermore, we express the position of the first perpendicular crack in terms of measurable material properties in Sec. IV] This result can be used to test our conjecture that the memory effect is caused by a plastic deformation. Section $\nabla$ is devoted to the summary and discussion. Technical details are summarized in Appendix [

\section{MODEL}

We propose the model of a viscoplastic material in a similar way as that demonstrated by Ooshida and Sekimoto [6]. We consider a viscoplastic material of thickness $H$ and width $2 L$ in a container, as shown in Fig. 11 The coordinate system $(x, z)$ is assumed such that the center of the container is at $x=0$ and the bottom is at $z=0$. For the mathematical simplicity, we restrict our attention to plane strain deformations of the viscoplastic material and we consider only a displacement $u(x, z, t)$ in the $x$ direction and a plastic strain $s(x, z, t)$, which express the occurrence of a plastic deformation. We assume that the 


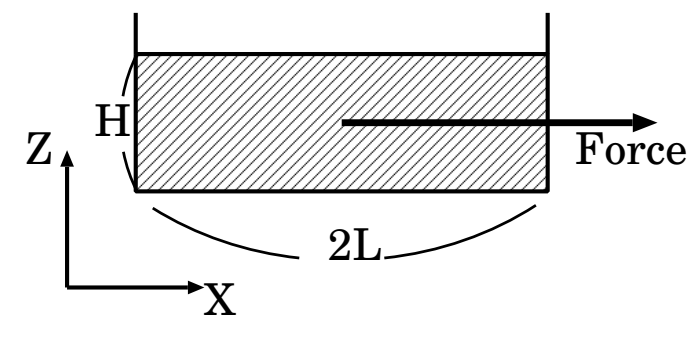

FIG. 1: The illustration of viscoplastic material in a container.

time evolutions of $u(x, z, t)$ and $s(x, z, t)$ are described as

$$
\begin{gathered}
\gamma \frac{\partial u(x, z, t)}{\partial t}=\frac{\partial \sigma_{x x}(x, z, t)}{\partial x}+\frac{\partial \sigma_{x z}(x, z, t)}{\partial z}+\alpha(t), \\
B \frac{\partial s(x, z, t)}{\partial t}=\left\{\begin{array}{cc}
0 & \left|\sigma_{x z}\right|<\sigma_{Y}(t) \\
\left(\left|\sigma_{x z}\right|-\sigma_{Y}(t)\right) \frac{\sigma_{x z}}{\left|\sigma_{x z}\right|} & \text { otherwise, }
\end{array}\right.
\end{gathered}
$$

where $\gamma$ is a coefficient of a viscosity, $\alpha(t)$ is an external force, $\sigma_{Y}(t)$ is a yield stress, $\sigma_{x x}(x, z, t)$ is a normal stress, $\sigma_{x z}(x, z, t)$ is a shear stress and $B$ is a coefficient which determines the speed of the plastic strain. $\sigma_{x x}(x, z, t)$ and $\sigma_{x z}(x, z, t)$ are determined by constitutive equations:

$$
\begin{gathered}
\sigma_{x x}(x, z, t)=(\lambda+2 \mu)\left(\frac{\partial u(x, z, t)}{\partial x}+c(t)\right), \\
\sigma_{x z}(x, z, t)=\mu\left(\frac{\partial u(x, z, t)}{\partial z}-s(x, z, t)\right),
\end{gathered}
$$

where $\lambda$ and $\mu$ are lame coefficients, and $c(t)$ is a reference strain rate. An increase in the reference strain rate represents an effect of shrinking by drying [7, []].

We consider the case where the functional forms of $\alpha(t), \sigma_{Y}(t)$, and $c(t)$ are given as

$$
\begin{gathered}
\alpha(t)=\left\{\begin{array}{cc}
\alpha_{M} \sin \left(\pi t / T_{1}\right) & t<T_{1} \\
0 & t>T_{1},
\end{array}\right. \\
\sigma_{Y}(t)=\left\{\begin{array}{cc}
\sigma_{Y 0} & t<T_{2} \\
\infty & t>T_{2},
\end{array}\right. \\
c(t)=\left\{\begin{array}{cc}
0 & t<T_{3} \\
b\left(t-T_{3}\right) & t>T_{3},
\end{array}\right.
\end{gathered}
$$

where $\alpha_{M}, \sigma_{Y 0}$, and $b$ represent the maximum value of an external force, the yield stress before drying, and the speed of drying, respectively.

In order to specify the model completely, we assume the boundary conditions. At the region where the material comes into contact with the container, the displacement is set to zero. In contrast, at the region where there is no contact between the material and the container, the stresses applied to the free surface become zero. Here, one peculiar phenomenon arises: when the material is dried, it peels off from the walls of the container. This phenomenon implies that the boundary conditions change when the peeling occurs. Hence, we assume boundary conditions

$$
\begin{array}{r}
u( \pm L, z, t)=0, \\
u(x, 0, t)=0, \\
\sigma_{x z}(x, H, t)=0,
\end{array}
$$

for $0<t<T_{2}$, and

$$
\begin{aligned}
\sigma_{x x}( \pm L, z, t) & =0, \\
u(x, 0, t) & =0, \\
\sigma_{x z}(x, H, t) & =0,
\end{aligned}
$$

for $t>T_{2}$, where we consider that the peeling occurs at $t=T_{2}$. In Table【 we summarize the functional forms of the parameters and the boundary conditions at the walls of the container when $T_{1}<T_{2}<T_{3}$.

Finally, we assume the condition of a crack formation. To the best of our knowledge, the condition has not yet been completely understood; however, two conditions have been used in previous works. These are the critical stress condition and the Griffith criterion. Under the critical stress condition, a crack is formed when the stress exceeds a material constant. This has been used in several numerical models [7] because of the technical advantage of the local condition. In contrast, under the Griffith criterion, a crack is formed when the energy released during the formation of a crack exceeds the increase of the surface energy. This condition has been used by Komatsu and Sasa in their theory 8]. Although we cannot determine which condition is more efficient, we employ the critical stress condition for the simplicity of the treatment. Concretely, we define an average normal stress at the position $x$ as

$$
\left\langle\sigma_{x x}(x, t)\right\rangle=\frac{1}{H} \int d z \sigma_{x x}(x, z, t) .
$$

Then, the condition of a crack formation is given as

$$
\left\langle\sigma_{x x}\left(x_{c}, t_{c}\right)\right\rangle=\sigma_{b} \rightarrow \sigma_{x x}\left(x_{c}, z, t\right)=0 \text { for } t>t_{c},
$$

where $x_{c}$ is the position of a crack, and $t_{c}$ is the time when the crack is formed. We only consider cracks that are perpendicular to the $x$ axis.

To summarize, our model consists of Eqs. (1) and (2) with parameters given by Eqs. (5), (6), and (7) under the boundary conditions (8) and (9), and the condition of a crack formation (11).

\section{A QUALITATIVE COMPREHENSION OF THE ORIGIN OF THE MEMORY EFFECT}

In order to understand how a viscoplastic material memorizes the influence of an external force that is applied before drying, we numerically calculate Eqs. (11) 
TABLE I: Boundary conditions (B.C.) and parameters

\begin{tabular}{|c|c|c|c|c|c|}
\hline & $t$ & $\alpha$ & $c$ & $\sigma_{Y}$ & B.C. at walls \\
\hline Applying an external force & $0<t<T_{1}$ & $\alpha_{M} \sin \left(\pi t / T_{1}\right)$ & 0 & $\sigma_{Y 0}$ & $u( \pm L, z, t)=0$ \\
\hline \multirow[t]{2}{*}{ Relaxation } & $T_{1}<t<T_{2}$ & 0 & 0 & $\sigma_{Y 0}$ & $u( \pm L, z, t)=0$ \\
\hline & $T_{2}<t<T_{3}$ & 0 & 0 & $\infty$ & $\sigma_{x x}( \pm L, z, t)=0$ \\
\hline Drying & $T_{3}<t$ & 0 & $b\left(t-T_{3}\right)$ & $\infty$ & $\sigma_{x x}( \pm L, z, t)=0$ \\
\hline
\end{tabular}

and (2) with the control parameter $\alpha_{M}$. In this numerical calculation, the initial conditions are given as $u(x, z, 0)=s(x, z, 0)=0$, and the parameter values are set as $H=1.0, L=10.0, \lambda=1.0, \mu=0.1, \sigma_{Y 0}=0.05$, $\sigma_{b}=0.01, \gamma=1.0, B=1.0, T_{1}=30, T_{2}=60, T_{3}=90$, and $b=0.00019$. In the following we report all quantities in reduced units, i.e., length in units of $H$, stress in units of $\lambda$, time in units of $\left(\gamma H^{2} / \lambda\right)$ and other quantities in units of the combinations of these units.

When no external force is applied to the material before drying, (that is, $\alpha_{M}=0$ ) stresses become zero at $t=T_{3}$ (just before starting drying process). Even when an external force is applied to a material, provided $\alpha_{M}$ is not so large that the shear stress exceeds the yield stress, the stresses remain zero at $t=T_{3}$. However, when $\alpha_{M}$ is sufficiently large, the stresses have a non-zero value at $t=T_{3}$, by a plastic deformation.

As an example, we show the results in the case $\alpha_{M}=$ 0.08 . When an external force $\alpha(t)$ increases in time from 0 , the material displaces to the direction of $x$, as shown in Fig. 2] Due to the influence of the boundary conditions $u( \pm L, z, t)=0$, the material is pulled in the left region $(x<0)$ and pushed in the right region $(x>0)$. When the

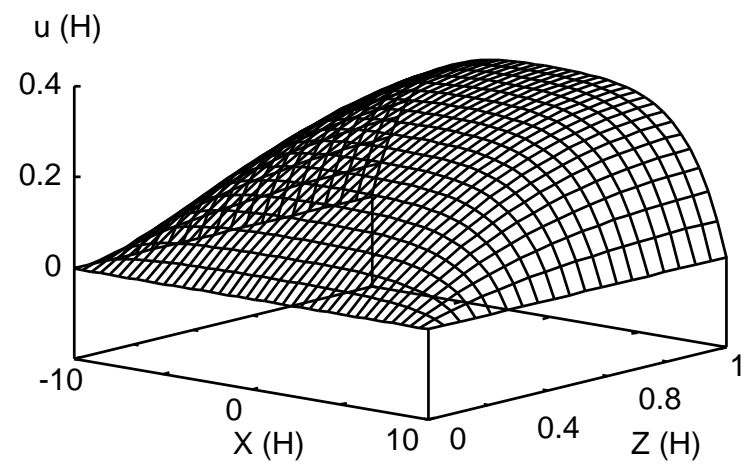

FIG. 2: $u\left(x, z, T_{1} / 2\right)$ as a function of $(x, z)$ in the case $\alpha_{M}=$ 0.08 .

deformation becomes sufficiently large, the shear stress $\sigma_{x z}(x, z, t)$ exceeds the yield stress $\sigma_{Y 0}$. Then, a plastic deformation occurs near the bottom $(z=0)$, as shown in Fig. 3. Due to this plastic deformation, the material remains pulled in the left region $(x<0)$ and pushed in the right region $(x>0)$ even after an external force $\alpha(t)$ becomes 0 . Therefore, the normal stress $\sigma_{x x}\left(x, z, T_{3}\right)$ is positive in the left region $(x<0)$ and negative in the

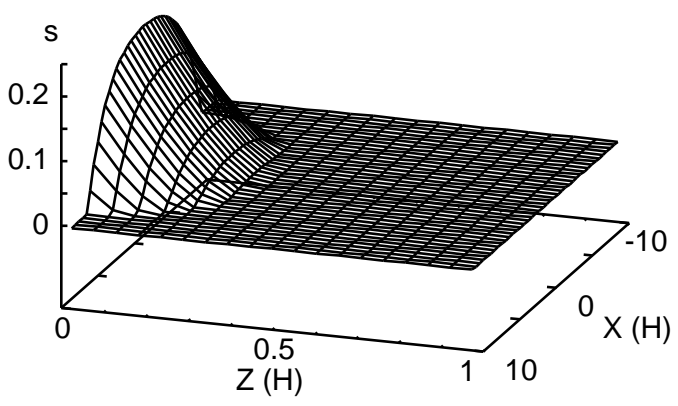

FIG. 3: $s\left(x, z, T_{3}\right)$ as a function of $(x, z)$ in the case $\alpha_{M}=$ 0.08 .

right region $(x>0)$, as shown in Fig. 4 In this manner,

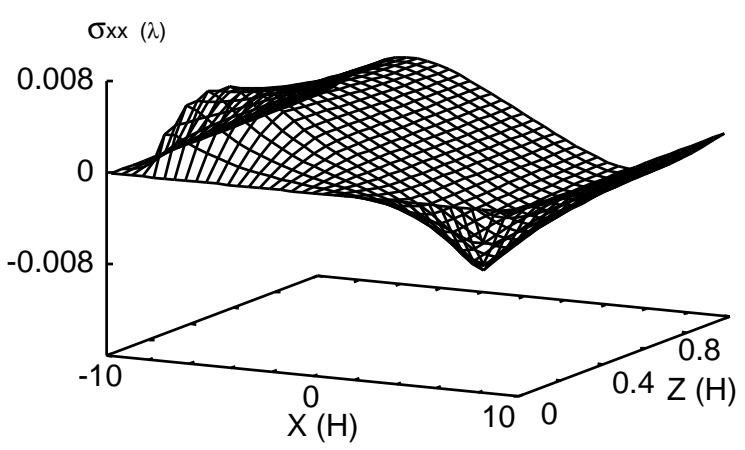

FIG. 4: $\sigma_{x x}\left(x, z, T_{3}\right)$ as a function of $(x, z)$ in the case $\alpha_{M}=$ 0.08 .

a material memorizes the effect of the force as a plastic deformation when an external force is applied to the material before drying.

Next, we investigate the influence of a plastic deformation on the formation of drying cracks by comparing the time evolutions of $\left\langle\sigma_{x x}(x, t)\right\rangle$ after starting the drying process $\left(t \geq T_{3}\right)$ in the cases $\alpha_{M}=0$ and $\alpha_{M}=0.08$, which are illustrated in Fig. 5] and Fig. 6] In the case $\alpha_{M}=0,\left\langle\sigma_{x x}(x, t)\right\rangle=0$ just before drying $\left(t=T_{3}\right)$; however, in the case $\alpha_{M}=0.08,\left\langle\sigma_{x x}(x, t)\right\rangle$ is positive in the left region $(x<0)$ and negative in the right region $(x>0)$ by a plastic deformation. As the material is dried, the average normal stress $\left\langle\sigma_{x x}(x, t)\right\rangle$ increases in 
a similar manner for both cases. However, until a crack is formed, the maximum values of $\left\langle\sigma_{x x}(x, t)\right\rangle$ in the case $\alpha_{M}=0.08$ are larger than those in the case $\alpha_{M}=0$. Due to this large stress, a crack is formed earlier in the case $\alpha_{M}=0.08$, than in the case $\alpha_{M}=0$.

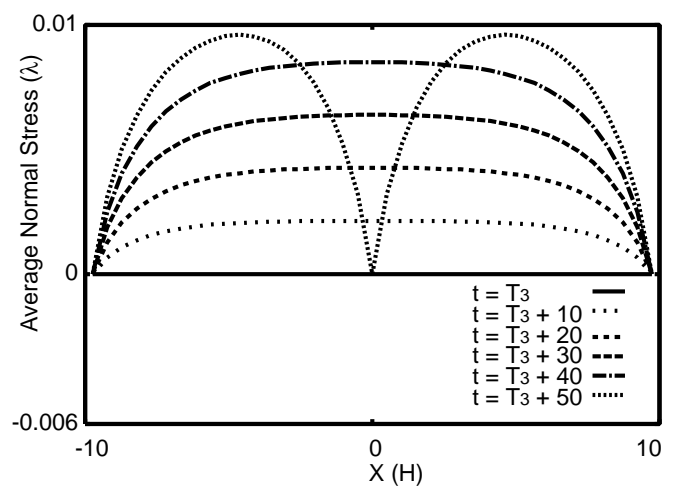

FIG. 5: The average normal stress $\left\langle\sigma_{x x}(x, t)\right\rangle$ in the case $\alpha_{M}=0$ as a function of $x$.

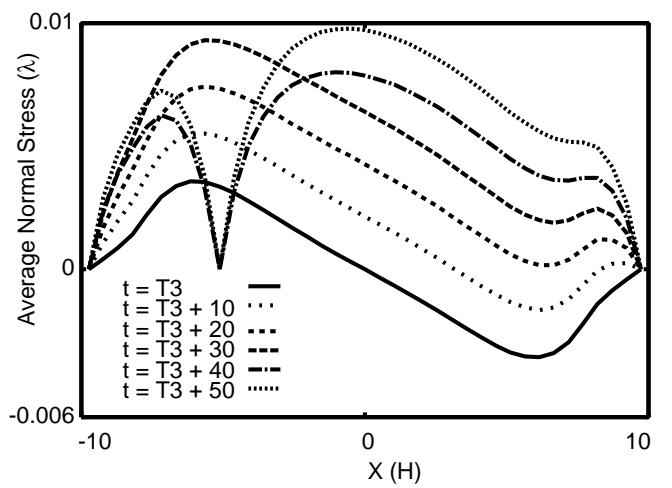

FIG. 6: The average normal stress $\left\langle\sigma_{x x}(x, t)\right\rangle$ in the case $\alpha_{M}=0.08$ as a function of $x$.

Here, we consider the correspondence between the results of the viscoplastic model and the experiments. In the viscoplastic model, we consider a crack that is perpendicular to an external force applied before drying. Due to the influence of a plastic deformation, the crack is formed earlier when an external force is applied to the material than when no force is applied. Therefore, we conjecture that in the experiments too, the perpendicular crack emerges due to the influence of a plastic deformation when an external force is applied to the material.

\section{QUANTITATIVE PREDICTIONS}

In the viscoplastic model, the position of the first perpendicular crack deviates from the center of the material to the opposite direction of an external force applied before drying as shown in Fig. 6] We wish to analytically express the position of the first perpendicular crack $x_{c}$ as a function of the maximum value $\alpha_{M}$ of the external force. However, since it is difficult to study the partial differential equations (11) and (2), we simplify the equations on some assumptions.

First, we assume that an external force $\alpha(t)$ and a reference strain rate $c(t)$ vary more slowly than the relaxation of a displacement $u(x, z, t)$ and a plastic strain $s(x, z, t)$. Then, it is sufficient to calculate the static solution of the equations, given an external force $\alpha(t)$, a yield stress $\sigma_{Y}(t)$, and a reference strain rate $c(t)$. Second, we discretize the partial derivative of $z$ as

$$
\begin{gathered}
\frac{\partial u(x, H, t)}{\partial z}=\frac{u(x, H, t)-u(x, 0, t)}{H}=\frac{u(x, H, t)}{H},(12) \\
\frac{\partial \sigma_{z}(x, H, t)}{\partial z}=\frac{\sigma_{x z}(x, H, t)-\sigma_{x z}(x, 0, t)}{H}=\frac{-\sigma_{x z}(x, 0, t)}{H},
\end{gathered}
$$

where $u(x, 0, t)=0$ and $\sigma_{x z}(x, H, t)=0$ (see Eqs. (8) and (9)). Based on these assumptions, the displacement at the surface $U(x, t)=u(x, H, t)$, the normal stress at the surface $T_{x x}(x, t)=\sigma_{x x}(x, H, t)$, and the shear stress at the bottom $T_{x z}(x, t)=\sigma_{x z}(x, 0, t)$ are determined by the equations

$$
\begin{gathered}
\frac{\partial T_{x x}(x, t)}{\partial x}-\frac{T_{x z}(x, t)}{H}+\alpha(t)=0, \\
T_{x x}(x, t)=(\lambda+2 \mu)\left(\frac{\partial U(x, t)}{\partial x}+c(t)\right), \\
T_{x z}(x, t)=\mu\left(\frac{U(x, t)}{H}-S(x, t)\right),
\end{gathered}
$$

where $S(x, t)$ is a plastic strain at the surface $(s(x, H, t))$. The time evolution of $S(x, t)$ is as follows: if

$$
\left|T_{x z}(x, t)\right|<\sigma_{Y}(t),
$$

$S(x, t)$ does not change in time. However, if

$$
\left|T_{x z}(x, t)\right|>\sigma_{Y}(t)
$$

$S(x, t)$ is determined by the condition

$$
\left|T_{x z}(x, t)\right|=\sigma_{Y}(t)
$$

which yields

$$
S(x, t)=\frac{U(x, t)}{H} \pm \frac{\sigma_{Y}(t)}{\mu}
$$

Here, the sign depends on the sign of $T_{x z}(x, t)$. By substituting Eqs. (15) and (16) into Eq. (14), we obtain the equation of $U(x, t)$ as

$$
(\lambda+2 \mu) \frac{\partial^{2} U}{\partial x^{2}}-\frac{\mu}{H^{2}} U+\frac{\mu}{H} S+\alpha(t)=0 .
$$


The functional forms of $\alpha(t), \sigma_{Y}(t)$, and $c(t)$ are given as Eqs. (5), (6), and (7), respectively. From Eqs. (8) and (9), the boundary conditions are rewritten as

$$
U(L, t)=U(-L, t)=0
$$

for $0<t<T_{2}$ and

$$
T_{x x}(L, t)=T_{x x}(-L, t)=0
$$

for $T_{2}<t$.

From Eq. (11), the condition of a crack formation is rewritten as

$$
T_{x x}\left(x_{c}, t_{c}\right)=\sigma_{b}
$$

Moreover, because $T_{x x}\left(x, t_{c}\right)$ has a maximum value at $x=x_{c}$, the equation

$$
\frac{\partial T_{x x}\left(x_{c}, t_{c}\right)}{\partial x}=0
$$

should be satisfied.

From these equations, $x_{c}$ can be calculated. The result is summarized below (see Appendix $\mathrm{A}$ for details of the calculation). First, we denote the threshold value of the external force by $\alpha_{Y 0}$, which is derived as

$$
\alpha_{Y 0}=\frac{\sigma_{Y 0} \cosh q L}{H(\cosh q L-1)},
$$

where

$$
q=\sqrt{\frac{\mu}{(\lambda+2 \mu) H^{2}}} .
$$

Then, if $\alpha_{M}<\alpha_{Y 0}$, the position of the first perpendicular crack $x_{c}$ is expressed as

$$
x_{c}=0 \text {. }
$$

In contrast, when $\alpha_{Y 0}<\alpha_{M}<\alpha_{Y 1}, x_{c}$ is determined by

$$
\begin{aligned}
\cosh q L= & B\left(0, x_{c}\right)+\frac{\sigma_{b} q H}{\sigma_{Y 0}-\alpha_{M} H} \sinh q x_{c} \\
& -\frac{D\left(L, x_{s}\right)}{\sinh q L}\left(1-\cosh q x_{c} \cosh q L\right),
\end{aligned}
$$

where $\alpha_{Y 1}$ is defined as

$$
\alpha_{Y 1}=\frac{\sigma_{Y 0}\left(2 \cosh q L-B\left(L, x_{s}\right)\right)}{\cosh q L-B\left(L, x_{s}\right)} .
$$

Here, $B\left(x_{1}, x_{2}\right)$ and $D\left(x_{1}, x_{2}\right)$ are defined as

$$
\begin{gathered}
B\left(x_{1}, x_{2}\right)=\cosh q\left(x_{1}-x_{2}\right)+q x_{2} \sinh \left(x_{1}-x_{2}\right), \\
D\left(x_{1}, x_{2}\right)=\sinh q\left(x_{1}-x_{2}\right)+q x_{2} \cosh q\left(x_{1}-x_{2}\right) .
\end{gathered}
$$

$x_{s}$ represents the region where a plastic deformation occurs as $-x_{s}<x<x_{s}$, which is determined by

$$
\alpha_{M} H+\left(\sigma_{Y 0}-\alpha_{M} H\right) B\left(L, x_{s}\right)=0 .
$$

We further extract a simple expression of $x_{c}$ by focusing on the region where $\alpha_{M}$ is adjacent to $\alpha_{Y 0}$. Assuming that

$$
q x_{c} \ll 1
$$

$$
q x_{s} \ll 1
$$

and expanding Eqs. (29) and (33) to the first order of $x_{c}$ and to the second order of $x_{s}$, we obtain

$$
x_{c}=-\frac{(\cosh q L-1)^{2}}{q^{2} \sigma_{b} \cosh q L}\left(\alpha_{M}-\alpha_{Y 0}\right) .
$$

In the calculation of $x_{c}$, we simplified the original partial differential equations based on some assumptions. In order to confirm the qualitative accuracy of Eqs. (28) and (36), we numerically calculate the solutions of $x_{c}$ for the original partial differential equations. In Fig. 7 and 8 we show the numerical solutions of $x_{c}$ for the case where the parameter values are same as in Sec. III (parameter set A) and for the case where the parameter values are set as $H=1.0, L=15.0, \lambda=1.0, \mu=0.2, \sigma_{Y 0}=0.03$, $\sigma_{b}=0.02, \gamma=1.0, B=1.0, T_{1}=300, T_{2}=600$, $T_{3}=500$, and $b=0.000005$ (parameter set B). There exists a threshold value $\alpha_{Y 0} . x_{c}$ remains zero when the external force $\alpha_{M}$ is smaller than the threshold value $\alpha_{Y 0}$ for both of the parameter sets. When the external force $\alpha_{M}$ is larger than the threshold value $\alpha_{Y 0}, x_{c}$ deviates from zero. In Fig. 9] we show the numerical solution of $x_{c}$ as a function of difference between the maximum value of an external force $\alpha_{M}$ and the threshold value $\alpha_{Y 0}$ for parameter set A. This figure clearly indicates $x_{c}$ deviates from zero as a linear function of the difference between $\alpha_{M}$ and $\alpha_{Y 0}$ when $\alpha_{M}$ is adjacent to $\alpha_{Y 0}$. These behavior qualitatively agree with Eqs. (28) and (36) though the values of $\alpha_{Y 0}$ are different.

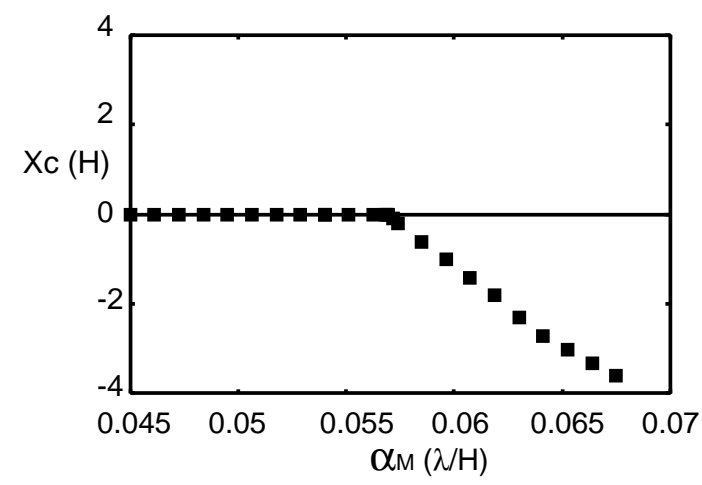

FIG. 7: The position of the first perpendicular crack $x_{c}$ as a function of the maximum value of an external force $\alpha_{M}$ for parameter set A.

Based on these results, we expect that in experiments too, the relation between the position of the first crack and the maximum value of an external force is expressed 


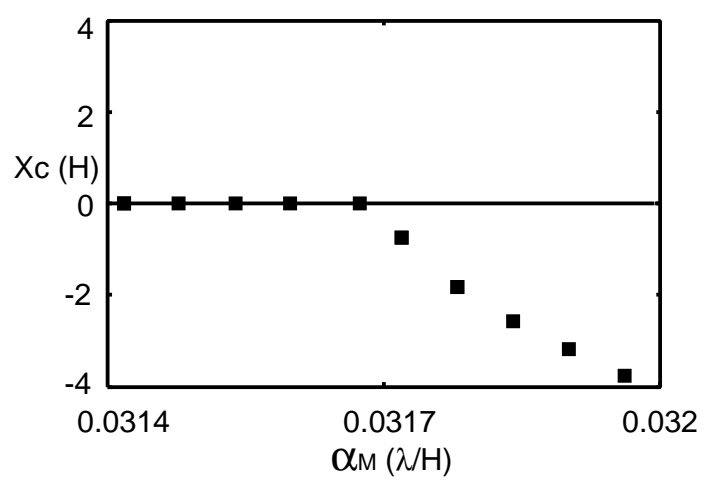

FIG. 8: The position of the first perpendicular crack $x_{c}$ as a function of the maximum value of an external force $\alpha_{M}$ for parameter set B.

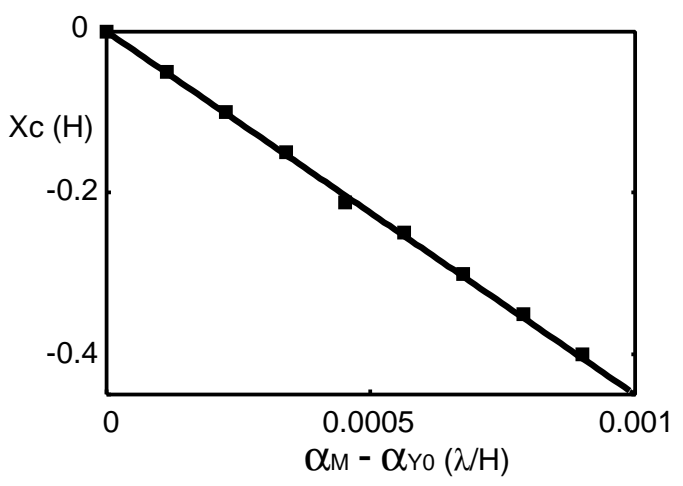

FIG. 9: The position of the first perpendicular crack $x_{c}$ as a function of difference between the maximum value of an external force $\alpha_{M}$ and the threshold value $\alpha_{Y 0}$ for parameter set A.

as Eqs. (28) and (36). The experimental confirmation of Eqs. (28) and (36) supports our conjecture that the memory effect on the formation of drying cracks arises from a plastic deformation of the material.

\section{SUMMARY AND DISCUSSION}

In this paper, we model the formation of drying cracks in a viscoplastic material. In numerical experiments, we observe that when an external force is applied before drying, a crack whose direction is perpendicular to the force emerges earlier than when no force is applied. This phenomenon occurs because of a plastic deformation. Based on this observation, we conjecture that a plastic deformation is the cause of the memory effect on the formation of drying cracks. In order to check this theory, we quantitatively predict the position of the first perpendicular crack. If Eqs. (28) and (36) are experimentally confirmed, it may be concluded that a plastic deformation causes the memory effect on the formation of drying cracks.

Here, we remark on the neglect of some quantities in our model. A displacement $w$ in the z-direction is not taken into account in our model. Moreover, normal stresses $\sigma_{x x}, \sigma_{y y}$ and $\sigma_{z z}$ are not taken into account in the equation which represents the occurrence of a plastic deformation, i.e., Eq. (2). The reason for neglecting $w$ is that $w$ plays little role in the formation of the crack because a crack is caused by a normal stress $\sigma_{x x}$ and $w$ hardly contributes to $\sigma_{x x}$. The reason for neglecting $\sigma_{x x}, \sigma_{y y}$ and $\sigma_{z z}$ is that we consider the situation where a plastic deformation occurs by a shear. To make sure that the neglect of these quantities does not affect our results, we simulated the model in which these quantities were taken into account. As far as we checked, we obtained qualitatively similar results with the model in present paper, for example, with regard to space distributions of a horizontal displacement $u(x, z, t)$, stresses $\sigma_{x x}(x, z, t)$ and $\sigma_{x z}(x, z, t)$ after applying an external force.

In addition, we remark on the the results for another choice of the parameters and the expressions of $\alpha(t)$, $\sigma_{Y}(t)$ and $c(t)$. As far as we checked, we observe the same phenomenon that the first perpendicular crack occurs earlier and the position of the crack deviates from center for any choice of the parameter as shown in Fig. 7 and Fig. 8 For any choice of the expressions of $\alpha(t), \sigma_{Y}(t)$ and $c(t)$, the same phenomenon are observed. Hence, we expect that our results are robust for the variation of the parameters and the expressions.

Recently, Nakahara and Matsuo measured the rheological property of a powder-water mixture $[9]$. From this measurement, they demonstrated that the mixture memorizes an external force before drying only when it behaves as a viscoplastic material with a finite yield stress. Moreover, they report that only when the strength of the force is larger than a threshold, the mixture memorizes the force. The relation between the memory of the force and a finite yield stress supports our conjecture that a plastic deformation plays an important role in the memory effect on the formation of drying cracks. Furthermore, the existence of the threshold coincides with the result of our model.

\section{Acknowledgments}

We thank A. Nakahara and Y. Matsuo for showing us experiments and for stimulating discussions. We also thank S. Sasa, T. S. Komatsu, and T. Ooshida for their valuable discussions.

\section{APPENDIX A: THE ANALYTICAL SOLUTION OF THE POSITION OF THE FIRST CRACK}

In this appendix, we show the path to obtain Eqs. (28) and (29), which determine the position of the first perpendicular crack $x_{c}$. This appendix consists of two sub- 
sections. In the first subsection, we calculate $S\left(x, T_{1}\right)$, and in the second subsection, we calculate $T_{x x}(x, t)(t>$ $\left.T_{3}\right)$. Substituting the expression of $T_{x x}(x, t)$ into Eqs. (24) and (25), we obtain Eqs. (28) and (29). In these calculations, we assume that $T_{1}<T_{2}<T_{3}$ in order to simplify the calculations.

\section{The calculation of $S\left(x, T_{1}\right)$}

First, we evaluate the minimum external force $\alpha_{Y 0}$, which yields a plastic deformation. Assuming that a plastic deformation does not occur $(S(x, t)=0)$ and solving Eq. (21) under the boundary condition (22), we obtain

$$
U(x, t)=\frac{\alpha(t) H^{2}}{\mu}\left(1-\frac{\cosh q x}{\cosh q L}\right),
$$

where

$$
q^{2}=\frac{\mu}{(\lambda+2 \mu) H^{2}} .
$$

By substituting Eq. (A1) into Eq. (16), we obtain a shear stress $T_{x z}(x, t)$ as

$$
T_{x z}(x, t)=\alpha(t) H\left(1-\frac{\cosh q x}{\cosh q L}\right) .
$$

The assumption that $S(x, t)=0$ is valid if the equation

$$
T_{x z}(x, t)<\sigma_{Y 0}
$$

is satisfied. This condition is equivalent to

$$
\alpha(t)<\alpha_{Y 0}
$$

where

$$
\alpha_{Y 0}=\frac{\sigma_{Y 0} \cosh q L}{H(\cosh q L-1)} .
$$

Hence, we find that if $\alpha_{M}<\alpha_{Y 0}$,

$$
S\left(x, T_{1}\right)=0 .
$$

If $\alpha_{M}>\alpha_{Y 0}$, a plastic deformation occurs. Then, assuming that a plastic deformation occurs in the region $-x_{s}<x<x_{s}$, we calculate $S\left(x, T_{1} / 2\right)$. From Eq. (20), $S(x, t)$ is given by

$$
S(x, t)=\left\{\begin{array}{cc}
0 & x<-x_{s} \\
\frac{U}{H}-\frac{\sigma_{Y 0}}{\mu} & -x_{s}<x<x_{s} \\
0 & x_{s}<x .
\end{array}\right.
$$

By substituting this into Eq. (21) and noting $\alpha\left(T_{1} / 2\right)=$ $\alpha_{M}$, we obtain the equations of $U\left(x, T_{1} / 2\right)$ as

$$
\left\{\begin{array}{cc}
(\lambda+2 \mu) \frac{\partial^{2} U}{\partial x^{2}}-\frac{\mu}{H^{2}} U+\alpha_{M}=0 & x<-x_{s} \\
(\lambda+2 \mu) \frac{\partial^{2} U}{\partial x^{2}}-\frac{\sigma_{Y 0}}{H}+\alpha_{M}=0 & -x_{s}<x<x_{s} \\
(\lambda+2 \mu) \frac{\partial^{2} U}{\partial x^{2}}-\frac{\mu}{H^{2}} U+\alpha_{M}=0 & x_{s}<x .
\end{array}\right.
$$

By solving these equations under the boundary conditions (22) and under the matching conditions that $U\left(x, T_{1} / 2\right), T_{x x}\left(x, T_{1} / 2\right)$, and $S\left(x, T_{1} / 2\right)$ are continuous at $x= \pm x_{s}$, we obtain the expression of $U\left(x, T_{1} / 2\right)$ and the equation to determine $x_{s}$. The expression of $U\left(x, T_{1} / 2\right)$ is

$$
U\left(x, T_{1} / 2\right)=\left\{\begin{array}{lc}
\frac{\alpha_{M} H^{2}}{\mu}+\frac{A_{s} H}{\mu} B\left(x,-x_{s}\right) & x<-x_{s} \\
\frac{A_{s} H}{2 \mu} q^{2}\left(x^{2}-x_{s}^{2}\right)+\frac{\sigma_{Y 0} H}{\mu}-x_{s}<x<x_{s} \\
\frac{\alpha_{M} H^{2}}{\mu}+\frac{A_{s} H}{\mu} B\left(x, x_{s}\right) & x_{s}<x,
\end{array}\right.
$$

where

$$
A_{s}=\sigma_{Y 0}-\alpha_{M} H
$$

and

$$
B\left(x_{1}, x_{2}\right)=\cosh q\left(x_{1}-x_{2}\right)+q x_{2} \sinh \left(x_{1}-x_{2}\right) .
$$

The equation to determine $x_{s}$ is

$$
\alpha_{M} H+A_{s} B\left(L, x_{s}\right)=0 .
$$

Substituting Eq. A10 into Eq. (A8), we obtain $S\left(x, T_{1} / 2\right)$ as

$$
S\left(x, T_{1} / 2\right)=\left\{\begin{array}{cc}
0 & x<-x_{s} \\
\frac{A_{s}}{2 \mu} q^{2}\left(x^{2}-x_{s}^{2}\right) & -x_{s}<x<x_{s} \\
0 & x_{s}<x .
\end{array}\right.
$$

Finally, $S\left(x, T_{1}\right)$ is calculated. First, we calculate $U\left(x, T_{1}\right)$ on the assumption that

$$
S\left(x, T_{1}\right)=S\left(x, T_{1} / 2\right) .
$$


Substituting Eq. (A15) into Eq. (21) and noting $\alpha(t)=\quad 0$, we obtain the equation of $U\left(x, T_{1}\right)$ as

$$
\left\{\begin{array}{cc}
(\lambda+2 \mu) \frac{\partial^{2} U}{\partial x^{2}}-\frac{\mu}{H^{2}} U=0 & x<-x_{s} \\
(\lambda+2 \mu) \frac{\partial^{2} U}{\partial x^{2}}-\frac{\mu}{H^{2}} U+\frac{A_{s}}{2 H} q^{2}\left(x^{2}-x_{s}^{2}\right)=0 & -x_{s}<x<x_{s} \\
(\lambda+2 \mu) \frac{\partial^{2} U}{\partial x^{2}}-\frac{\mu}{H^{2}} U=0 & x_{s}<x .
\end{array}\right.
$$

By solving this equation under the boundary conditions and the matching conditions, we obtain $U\left(x, T_{1}\right)$ as

$$
U\left(x, T_{1}\right)=\left\{\begin{array}{cc}
\frac{A_{s} H}{\mu}\left(B\left(x,-x_{s}\right)-B\left(L, x_{s}\right) \frac{\cosh q x}{\cosh q L}\right) & x<-x_{s} \\
\frac{A_{s} H}{\mu}\left(1+\frac{q^{2}\left(x^{2}-x_{s}^{2}\right)}{2}-B\left(L, x_{s}\right) \frac{\cosh q x}{\cosh q L}\right) & -x_{s}<x<x_{s} \\
\frac{A_{s} H}{\mu}\left(B\left(x, x_{s}\right)-B\left(L, x_{s}\right) \frac{\cosh q x}{\cosh q L}\right) & x_{s}<x .
\end{array}\right.
$$

Substituting this equation and Eq. (A15) into Eq. (16), we get $T_{x z}\left(x, T_{1}\right)$ as

$$
T_{x z}\left(x, T_{1}\right)=\left\{\begin{array}{cc}
A_{s}\left(B\left(x,-x_{s}\right)-B\left(L, x_{s}\right) \frac{\cosh q x}{\cosh q L}\right) & x<-x_{s} \\
A_{s}\left(1-B\left(L, x_{s}\right) \frac{\cosh q x}{\cosh q L}\right) & -x_{s}<x<x_{s} \\
A_{s}\left(B\left(x, x_{s}\right)-B\left(L, x_{s}\right) \frac{\cosh q x}{\cosh q L}\right) & x_{s}<x .
\end{array}\right.
$$

The assumption that $S\left(x, T_{1}\right)=S\left(x, T_{1} / 2\right)$ is valid when the equation

$$
\left|T_{x z}\left(x, T_{1}\right)\right|<\sigma_{Y 0}
$$

is satisfied, because $S\left(x, T_{1}\right)=S\left(x, T_{1} / 2\right)$ implies that a plastic deformation does not occur for $T_{1} / 2<t<T_{1}$. This condition is equivalent to

$$
\alpha_{M}<\alpha_{Y 1},
$$

where

$$
\alpha_{Y 1}=\frac{\sigma_{Y 0}\left(2 \cosh q L-B\left(L, x_{s}\right)\right)}{\cosh q L-B\left(L, x_{s}\right)} .
$$

Hence, if

$$
\alpha_{Y 0}<\alpha_{M}<\alpha_{Y 1}
$$

then $S\left(x, T_{1}\right)$ is expressed by Eq. A15).

\section{The calculation of $T_{x x}(x, t)$ for $t>T_{3}$}

In this subsection, we calculate $T_{x x}(x, t)$ for $t>T_{3}$ in the cases $\alpha_{M}<\alpha_{Y 0}$ and $\alpha_{Y 0}<\alpha_{M}<\alpha_{Y 1}$.

If $\alpha_{M}<\alpha_{Y 0}$, then $S\left(x, T_{1}\right)=0$. Since $\sigma_{Y}=\infty$, $S(x, t)=0$ for $t>T_{3}$. Substituting $S(x, t)=0$ and $\alpha(t)=0$ into Eq. (21), we obtain the equation of $U(x, t)$ for $t>T_{3}$ as

$$
(\lambda+2 \mu) \frac{\partial^{2} U}{\partial x^{2}}-\frac{\mu}{H^{2}} U=0 .
$$

By solving this equation under the boundary conditions (23), we obtain $U(x, t)$ for $t>T_{3}$ as

$$
U(x, t)=-\frac{c(t) \sinh q x}{q \cosh q L} .
$$


Substituting this into Eq. (15), we get $T_{x x}(x, t)$ in the case $\alpha_{M}<\alpha_{Y 0}$ as

$$
T_{x x}(x, t)=(\lambda+2 \mu) c(t)\left(1-\frac{\cosh q x}{\cosh q L}\right) .
$$

If $\alpha_{Y 0}<\alpha_{M}<\alpha_{Y 1}, S\left(x, T_{1}\right)$ is expressed by Eq. A15. Since $\sigma_{Y}=\infty$,

$$
S(x, t)=S\left(x, T_{1}\right)
$$

for $t>T_{3}$, where $S\left(x, T_{1}\right)$ is expressed by Eq. A15. Substituting Eq. (A26) into Eq. (21) and noting $\alpha(t)=$ 0 , we obtain the same equation of $U(x, t)$ as Eq. A16. By solving this equation under the boundary conditions (23) and the matching conditions, we obtain $U(x, t)$ for $t>T_{3}$ as

$$
U(x, t)=\left\{\begin{array}{lc}
-\frac{c(t) \sinh q x}{q \cosh q L}+\frac{A_{s} H}{\mu}\left(B\left(x,-x_{s}\right)-D\left(L, x_{s}\right) \frac{\cosh q x}{\sinh q L}\right) & x<-x_{s} \\
-\frac{c(t) \sinh q x}{q \cosh q L}+\frac{A_{s} H}{\mu}\left(1+\frac{q^{2}\left(x^{2}-x_{s}^{2}\right)}{2}-D\left(L, x_{s}\right) \frac{\cosh q x}{\sinh q L}\right) & -x_{s}<x<x_{s} \\
-\frac{c(t) \sinh q x}{q \cosh q L}+\frac{A_{s} H}{\mu}\left(B\left(x, x_{s}\right)-D\left(L, x_{s}\right) \frac{\cosh q x}{\sinh q L}\right) & x_{s}<x,
\end{array}\right.
$$

where

$$
D\left(x_{1}, x_{2}\right)=\sinh q\left(x_{1}-x_{2}\right)+q x_{2} \cosh q\left(x_{1}-x_{2}\right) .
$$

Substituting this into Eq. (15), we get $T_{x x}(x, t)$ for $t>T_{3}$ in the case $\alpha_{Y 0}<\alpha_{M}<\alpha_{Y 1}$ as

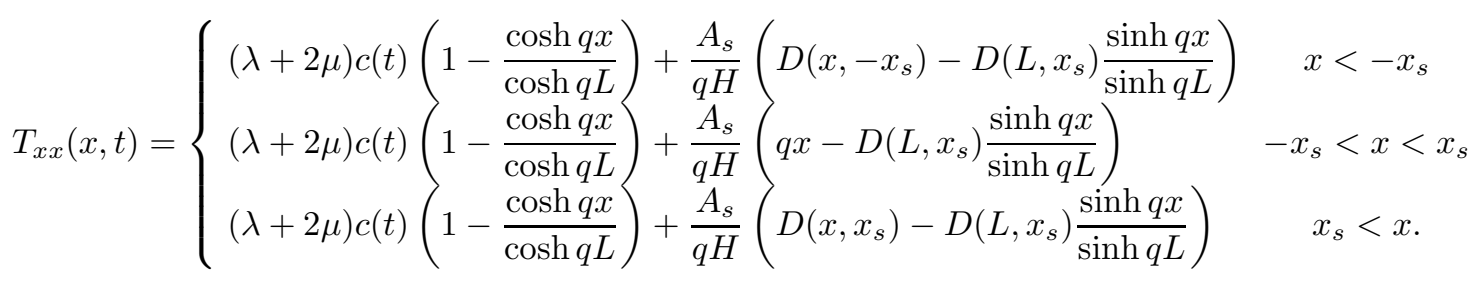

[1] G. Groisman and E. Kaplan, Europhys. Lett 25, 415 (1994).

[2] A. Nakahara and Y. Matsuo, Bussei Kenkyuu 81, 2 (2003).

[3] L. Vanel, D. Howell, D. Clark, R. P. Behringer and E. Clement, Phys. Rev. E 60, R5040 (1999).

[4] M. Cloitre, R. Borrega and L. Leibler, Phys. Rev. Lett. 85, 4819 (2000).

[5] Y. Miyamoto, K. Fukao, H. Yamao and K. Sekimoto,
Phys. Rev. Lett. 88, 225504 (2002).

[6] T. Ooshida and K. Sekimoto, cond-mat/0410306

[7] S. Kitsunezaki, Phys. Rev. E 60, 6449 (1999).

[8] T. S. Komatsu and S. Sasa, Jpn. J. Appl. Phys. 36, 391 (1997).

[9] A. Nakahara and Y. Matsuo, J. Phys. Soc. Jpn. 74, 1362 (2005). 